\title{
Challenges and Requirements for Introducing Impairment-Awareness into the Management and Control Planes of ASON/GMPLS WDM Networks
}

\author{
Ricardo Martínez and Carolina Pinart, Centre Tecnològic de Telecomunicacions de Catalunya (CTTC) \\ Filippo Cugini, CNIT \\ Nicola Andriolli, Luca Valcarenghi, and Piero Castoldi, Scuola Superiore Sant'Anna \\ Lena Wosinska, Royal Institute of Technology (KTH/ICT) \\ Jaume Comellas and Gabriel Junyent, Universitat Politècnica de Catalunya (UPC)
}

\begin{abstract}
The absence of electrical regenerators in transparent WDM networks significantly contributes to reduce the overall network cost. In transparent WDM networks, a proper resource allocation requires that the presence of physical impairments in Routing and Wavelength Assignment (RWA) and lightpath provisioning be taken into account. In this article a centralized, a hybrid centralized-distributed and two distributed approaches that integrate information about most relevant physical impairments in RWA and lightpath provisioning are presented and assessed. Both centralized and hybrid approaches perform a centralized path computation at the management-plane level, utilizing physical impairment information, while the lightpath provisioning is done by the management plane or the control plane, respectively. The distributed approaches fall entirely within the scope of the ASON/GMPLS control plane. For these two approaches, we provide functional requirements, architectural functional blocks, and protocol extensions for implementing either an impairment-aware real-time RWA, or a lighpath provisioning based on impairment-aware signaling.
\end{abstract}

\section{INTRODUCTION}

Wavelength Division Multiplexing (WDM) is a subfiber multiplexing technique that can efficiently accommodate various data traffic flows (IP, SONET-SDH, Ethernet, etc.) at the optical layer. Originally, the provisioning problem in WDM networks was solved at the management plane (MP) level by an operator-driven centralized approach. More recently, the introduction of the optical control plane (CP) allowed the simplification and automatation of provisioning in WDM networks. In the context of wavelengthrouted networks, the ITU-T Automatic Switched Optical Network (ASON) standard [1] describes the set of CP components that are to be used to manipulate transport network resources in order to provide the functionality of setting up, maintaining, and releasing optical connections. Generalized Multiprotocol Label Switching (GMPLS) is an implementation of the CP that has been developed by the IETF to facilitate the establishment of label switched paths (LSPs) [2], involving signaling, routing, and resource management functions and protocols. GMPLS implements all functional entities necessary for controlling an ASON, actually going beyond the pure optical domain and being capable of setting up LSPs in a variety of data plane technologies.

The feasibility of relevant signal processing functions (e.g., amplification, filtering, and dispersion compensation) of optical transmission systems and the availability of network nodes capable of routing and switching in the optical domain has given rise to the introduction of highly flexible and transparent optical network solutions that eliminate the restrictions of periodic electrical-signal regeneration to overcome. Circuit-switched optical networks will be able to support all-optical connections at the wavelength granularity that can transparently traverse network nodes. The main advantage of all-optical networks is their transparency to bit rate and traffic format. However, due to the lack of elec- 
trical regeneration and to the evolution from SONET/SDH point-to-point to optical signals being routed, added/dropped, multiplexed, and demultiplexed in the optical layer, optical-signal impairments may accumulate along an optical connection. Accordingly, if GMPLS is to be employed in the control plane of an all-optical network, it should take into account physical impairments when performing routing and wavelength assignment (RWA) and the subsequent provisioning.

Therefore, the objective of considering physical impairments when establishing optical connections is to assure sufficient signal quality to avoid potential degradations of the quality of service (QoS) required by the connection request. QoS is typically defined as the performance specification of a communication system and is usually represented quantitatively by performance parameters that may involve several layers of the OSI model. The scenario of this article is that of a transparent domain, in which optical connections traverse all-optical devices and amplified fiber segments. Thus, the term QoS is applied to the physical layer of the transparent network (layer 1 in the IP network architecture), that is, it refers to the quality of the optical signal in the WDM links and at the (optical) receiving end. Then, QoS is quantified with physical-layer parameters such as optical signalto-noise ratio (OSNR), optical power, or end-toend latency. We assume that full retiming, reshaping, and reamplification (3R) of optical channels is performed at the edges of transparent domains. For the considered all-optical scenario, we propose requirements and solutions for performing impairment-aware RWA when provisioning optical connections with stringent requirements on signal quality. Two families of approaches are described:

- In the first one, the RWA is centralized while the wavelength provisioning can be either centralized or distributed.

- The second one represents a distributed approach in which both RWA and provisioning are based on GMPLS protocol suite extensions.

For all schemes, centralized operations are typically done at the MP level while distributed operations are run at the CP level.

The rest of the article is organized as follows. We describe the most relevant physical impairments and performance measurements in transparent WDM networks. We present centralized and distributed approaches to account for physical impairments in the lightpath setup process. We also discuss the advantages and drawbacks of these approaches. We elaborate on the possible implementations of an impairment-aware GMPLS-based distributed control plane. Finally, we draw conclusions.

\section{PHYSICAL IMPAIRMENTS IN TRANSPARENT WDM NETWORKS}

Current SONET/SDH networks are opaque, that is, optical signals are converted into the electrical domain, regenerated, and converted back to the optical domain at each hop. In other words, fiber links are isolated analog systems, and spectral impairments can be solved at each node by regenerating the signals using electronics. Opto-electronic conversion also enables in-service monitoring of transmission quality through evaluating errors in SDH headers, such as block or bit-interleaved errors. On the other hand, in dynamic transparent networks, network nodes will be able to establish end-to-end connections upon request, totally within the optical domain. These optical connections are referred to as lightpaths. The main advantages of transparent networks are high throughput and freedom in choosing data rates and formats, as well as significant cost reduction due to no need for signal processing at each network node. However, as a consequence of the lack of signal regeneration, the main problem can be the accumulation of impairments (noise, cross-talk, dispersion, and fiber nonlinearities), as depicted in Fig. 1a.

There are two main alternatives to obtain information about physical impairments: modeling and real-time monitoring. In the literature we may find models for some performance parameters that aim at capturing the most dominant impairments in high-speed networks, such as the polarization mode dispersion (PMD) and OSNR constraint models proposed by Huang et al. [3]. On the other hand, recent advances in optical monitoring techniques make it possible to obtain Optical Performance Monitoring (OPM) parameters (e.g., optical power per channel and aggregate optical power, wavelength drift, channel OSNR, and in-band OSNR) in milliseconds and in a nonintrusive way, that is, through extracting a portion of WDM signals by tapping optical fibers.

Physical impairments can be captured by monitoring a number of performance parameters. Performance monitoring traditionally refers to monitoring at the SONET/SDH (electrical) layer for bit/block error rates and other service quality (QoS) measures, whereas OPM involves the monitoring of physical-layer performance, not necessarily correlated with the electrical performance. However, much effort has been made in identifying OPM techniques that can be used for evaluating QoS applications [4], as well as spectral "substitutes" for BER measurement, such as optical Q-meters. The topic of OPM has been discussed in the literature since the early 1990s and the term itself has taken on multiple definitions. In this work, we adopt a broad definition of OPM: "physical-layer monitoring of the signal quality for the purpose of determining the health of the signal in the optical domain." OPM can be seen as composed of two different parts. The first part is WDM channel-layer monitoring, which involves a determination of the opticaldomain characteristics essential for transport and channel management at the WDM layer. This part includes the real-time measurements of channel presence, wavelength drift, power levels, and the spectral OSNR. The second part of OPM involves monitoring the data protocol information, and includes digital measurements such as the BER, when used to infer properties of the analog optical signal. Note that this part deals with protocol-performance monitoring,
Recent advances in

optical monitoring

techniques make it

possible to obtain

OPM parameters

(e.g., optical power

per channel and

aggregate optical

power, wavelength

drift, channel OSNR,

and in-band OSNR)

in milliseconds and in

a nonintrusive way. 


Both modeling and
real-time OPM
provide information
on a per channel
basis, which shall be
matched to the
needs of lightpath
computation, usually
done on a hop (link)
basis. Therefore, to
estimate the quality
of a lightpath it is
necessary to
aggregate physical
impairments
expressed by one or
more link-state
parameters.

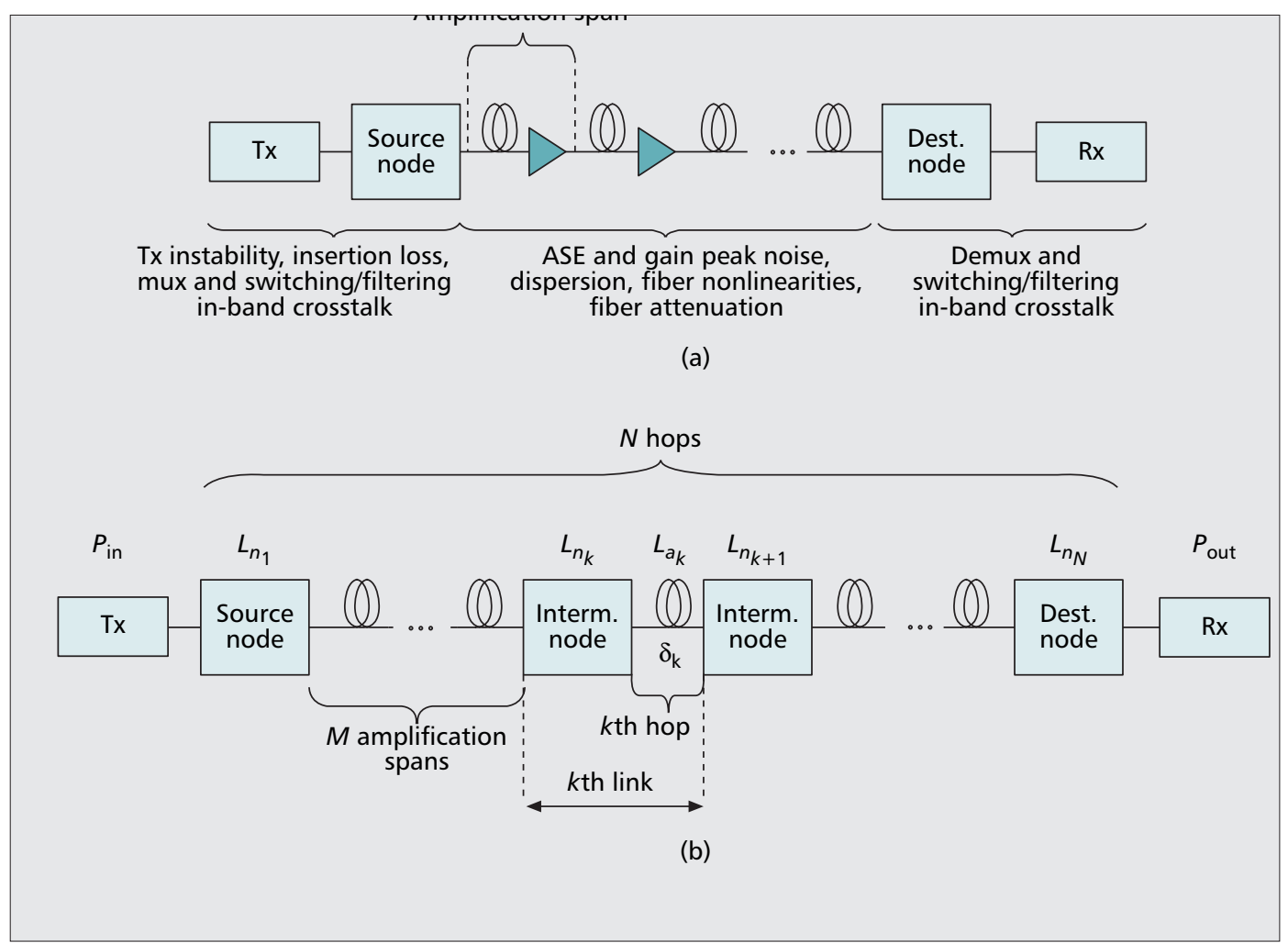

Figure 1. Transparent lightpath: a) degradation; b) link model. and therefore, unlike in the first part of OPM, some measures may be protocol-dependent. In 2004 the ITU-T defined a list of OPM parameters that can be used for impairment-aware RWA [5]. Note that both modeling and realtime OPM provide information on a per channel basis, which shall be matched to the needs of lightpath computation, usually done on a hop (link) basis. Therefore, to estimate the quality of a lightpath it is necessary to aggregate physical impairments expressed by one or more link-state parameters.

Impairment-aware RWA assumes that the quality of an optical signal traversing $N$ hops can be estimated as a function (maximum, minimum, addition, etc.) of the impairments introduced by the optical elements along the path. Figure $1 \mathrm{~b}$ illustrates a possible model for an $N$ hop lightpath, where the $k$-th link is modeled as the aggregate of all impairments caused by the $k$-th optical node and the amplification spans between the $k$-th and $(k+1)$-th nodes. One of the most important physical impairments is dispersion. The main contributions to dispersion are chromatic dispersion and PMD. Chromatic dispersion is deterministic in nature, and can be easily compensated in the optical links, especially in reasonable linear systems. On the contrary, PMD is a stochastic, timevarying effect that can be modeled statically but is harder to compensate in practice. PMD influences system performance in high-speed transmissions (i.e., above $10 \mathrm{~Gb} / \mathrm{s}$ ) and can be usually overcome by not exceeding a maximum fiber length that assures PMD compensation. In Fig. $1 \mathrm{~b}, \delta_{k}$ is the PMD constraint in the RWA for each link, defined as the maximum tolerated pulse broadening, and it depends on the bit rate, the PMD parameter of each of the fiber segments in the link $\left(D_{P M D}\right)$, and the length of the segments (l) [6]. In Fig. 1b it is assumed that chromatic dispersion is compensated while PMD is not.

Noise coming from amplified spontaneous emission (ASE) is a dominant impairment at any bit rate, which cannot be compensated. ASE noise can be measured or estimated via OSNR values and/or individual power levels of WDM signals and noise. The power received $\left(P_{\text {out }}\right)$ can be obtained as a function of laser source power $\left(P_{\text {in }}\right.$ in Fig. $\left.1 \mathrm{~b}\right)$ or OSNR $\left(P_{\text {in }} / N_{\text {in }}\right)$, and the insertion losses, cross-talk, attenuation, and gains of the elements along the $N$ hops. In the $k$-th link, $L_{n k}$ captures the insertion loss of the node, as well as the cross-talk of mux/dmux and filters/switches. $L_{a k}$ is a function of the fiber attenuation $(a)$ and amplification gain $\left(G_{a}\right)$ in each of the $M$ spans. We consider that the noise received $\left(N_{\text {out }}\right)$ consists of ASE noise, which in EDFAs basically depends on $G_{a}$, the amplifier's spontaneous emission factor $\left(n_{s p}\right)$, the channel's frequency, and the optical bandwidth of the system.

It is worth noting that BER is one of the major parameters of the signal quality because it captures all the impairments along the path. However, in a transparent domain BER can only be measured at the receiving edge, denoted as $\mathrm{Rx}$ in Fig. 1. Thus, on the link basis, BER can only be estimated (not measured), for example, by using OSNR, as proposed in [3]. For this reason, Fig. 1b does not include BER in the link model. Finally, we assume that the optical network is designed to avoid excessive nonlinear 


\begin{tabular}{|c|c|c|}
\hline$k$ th link parameter & Description & Physical link constraint \\
\hline Node's insertion losses, $L_{n k}$ & $\begin{array}{l}\text { Captures the insertion loss of the node, and the } \\
\text { crosstalk of mux/demux and filters/switches }\end{array}$ & OSNR constraint \\
\hline Noise power, $N_{k}$ & $\begin{array}{l}\text { Consists of ASE noises, that is: } \\
\text { - Amplification gain }\left(G_{a}\right) \\
\text { - Amplifier's emission factor }\left(n_{s p}\right) \\
\text { - Channel's frequency } \\
\text { - Optical bandwidth }\end{array}$ & OSNR constraint \\
\hline Link length, I & Represents the fiber length & PMD constraint \\
\hline Total loss of the link, $L_{a k}$ & $\begin{array}{l}\text { Represents the total loss of the } k \text { th link, that is, } \\
\text { a function of the total fiber attenuation }\left(a_{k}\right) \text { and } \\
\text { the amplificiation gain }\left(G_{a}\right) \text { in each of the } M \\
\text { amplification spans }\end{array}$ & OSNR constraint \\
\hline link PMD parameter, $D_{P M D k}$ & Represents the fiber PMD parameter & PMD constraint \\
\hline
\end{tabular}

The NMS approach

consists of the

classical architecture

adopted by the

optical transport

networks (i.e., the

telecommunications

management

network). The NMS

is responsible for the

administration,

computation, and

provisioning

functionalities of the

whole network. impairments (e.g., four-wave mixing, cross-phase and self-phase modulations, etc.) in the transparent routes; therefore, including nonlinear impairments in our model is not within the scope of this article. Although nonlinear impairments can impose significant limitations on both high-speed channel provisioning and very dense DWDM transport capacity, the above assumption is motivated by two main reasons. As stated in [6], considering nonlinear impairments in RWA would require detailed knowledge of the entire physical infrastructure and current loading of each optical signal on every link, because of the complex dependencies that nonlinear impairments create among routes. Moreover, no generic analytical model of nonlinear impairments has been derived [3] that allows handling these effects as constraints in impairment-aware RWA. For these reasons, the effects of nonlinear impairments are handled by increasing the required OSNR level at the receiving end by a link budget margin. However, this approximation may lead to either an overestimation or an underestimation of the real impact of the nonlinear impairment effect, and thus it is not considered here.

\section{ACCOUNTING FOR PhYSICAL IMPAIRMENTS IN LIGHTPATH SET UP}

Table 1 illustrates the relevant link parameters according to the model depicted in Fig. 1. Besides these physical link attributes, different service classes can be defined specifying the required signal-quality (i.e., QoS) that the lightpath must satisfy in terms of BER (estimated from OSNR [3]) and higher-layer QoS requirements (e.g., latency). They will serve as thresholds for deciding whether a particular link (i.e., link constraints) is acceptable by the RWA algorithm. The insertion of this intelligence in the optical layer can be accomplished through two different approaches: centralized and dis- tributed. In the former, all paths to be established within a domain are computed and/or established by a single and centralized network element (e.g., network management system (NMS) or path computation element (PCE), [7]). In the latter, a distributed and intelligent optical control plane (OCP) embedded in each network element (NE) is responsible for both route computation and lightpath establishment (Fig. 4).

\section{Centralized Model}

In the centralized model a single element, which is reachable by all the NEs within the transparent domain, plays the role of a server wherein all the connections to be established are served [3]. For this purpose, this entity is aware of the complete network topology, resource availability, and physical parameters in a central repository termed the Traffic Engineering Database (TED), as shown in Fig. 2a. Thus, in this model the performance monitoring system updates the TED information with the complete set of performance parameters within the entire domain, referred to as $\mathrm{S}$, which is then considered by the routing computation process. By doing so, this central element is able to guarantee and satisfy the specific set of lightpath requirements (e.g., bandwidth, diversity, optical signal quality, latency, etc.) requested by a particular NE.

Two different approaches are considered to build the centralized model: using the traditional NMS agent and the PCE entity.

The NMS approach consists in the classical architecture adopted by the optical transport networks (i.e., the Telecommunications Management Network). The NMS is responsible for the administration, computation, and provisioning functionalities of the whole network. As shown in Fig. 3, which illustrates the flow diagram of different impairment-aware lightpath setup approaches, once the connection request is received by the NMS, the routing computation 


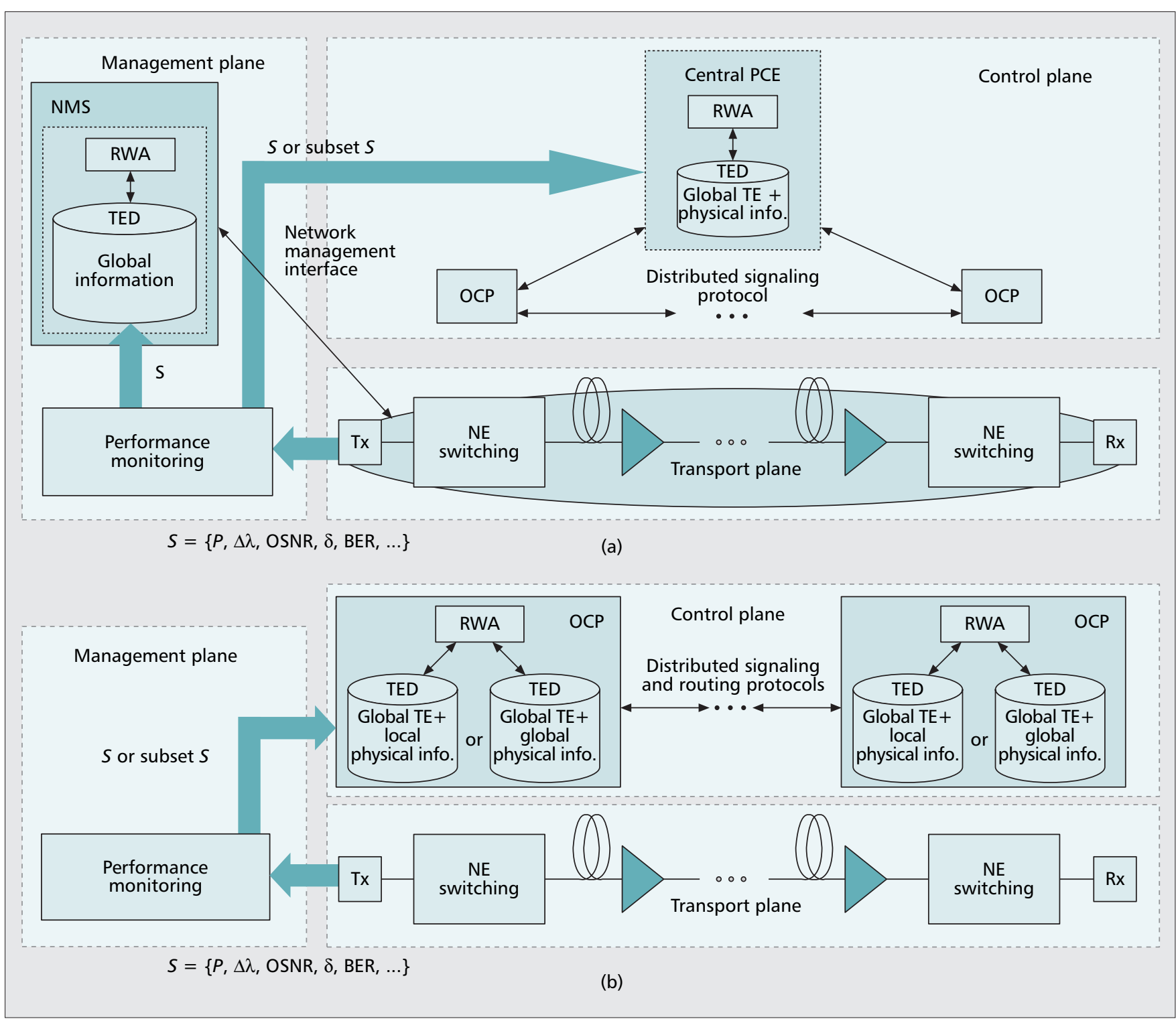

Figure 2. Structure of the RWA and TED scenarios for the a) centralized; b) distributed models.

process is launched considering both the current TED information and the received set of requirements of the connection request (step A1). Then, the NMS configures all the NE's optical switching equipment (e.g., optical crossconnects) involved in the path in a parallel way (using the network management interface) to set up the lightpath (step A2).

The PCE-based approach is actually hybrid because, differently from the NMS-based approach, it also utilizes a reduced set of control plane functionalities (i.e., distributed signaling) to set up all the paths. In such an approach, all OCP modules send queries to the central PCE element, with which all of them can communicate for route computation issues. Whenever the PCE receives a path computation request, it computes the required path, taking into account the TED information and the on-demand connection requirements (step B1). Then, the computed route is converted into an explicit route (i.e., a set of nodes and links) which is returned to the source OCP for establishing the lightpath by means of a distributed signaling protocol (steps B2-B6).

\section{Distributed ApPROACHES}

In this model each NE's OCP is responsible to setup lightpaths within the transparent domain (Fig. 2b). For this purpose, a common and distributed GMPLS-based control plane manages the required procedures for establishing end-toend lightpaths (i.e., routing and signaling). We propose two different approaches under the distributed model: distributed routing and distributed signaling.

In the distributed routing approach, some extensions to current GMPLS routing protocols (e.g., open shortest path first, OSPF-TE) need to be implemented with the aim of carrying physical impairment information besides traffic engineering (TE) attributes, such as unreserved bandwidth. Thus, a global TED is built at each OCP (Fig. 2b), which maintains an up-to-date picture of both resource utilization and physical parameters concerning any 


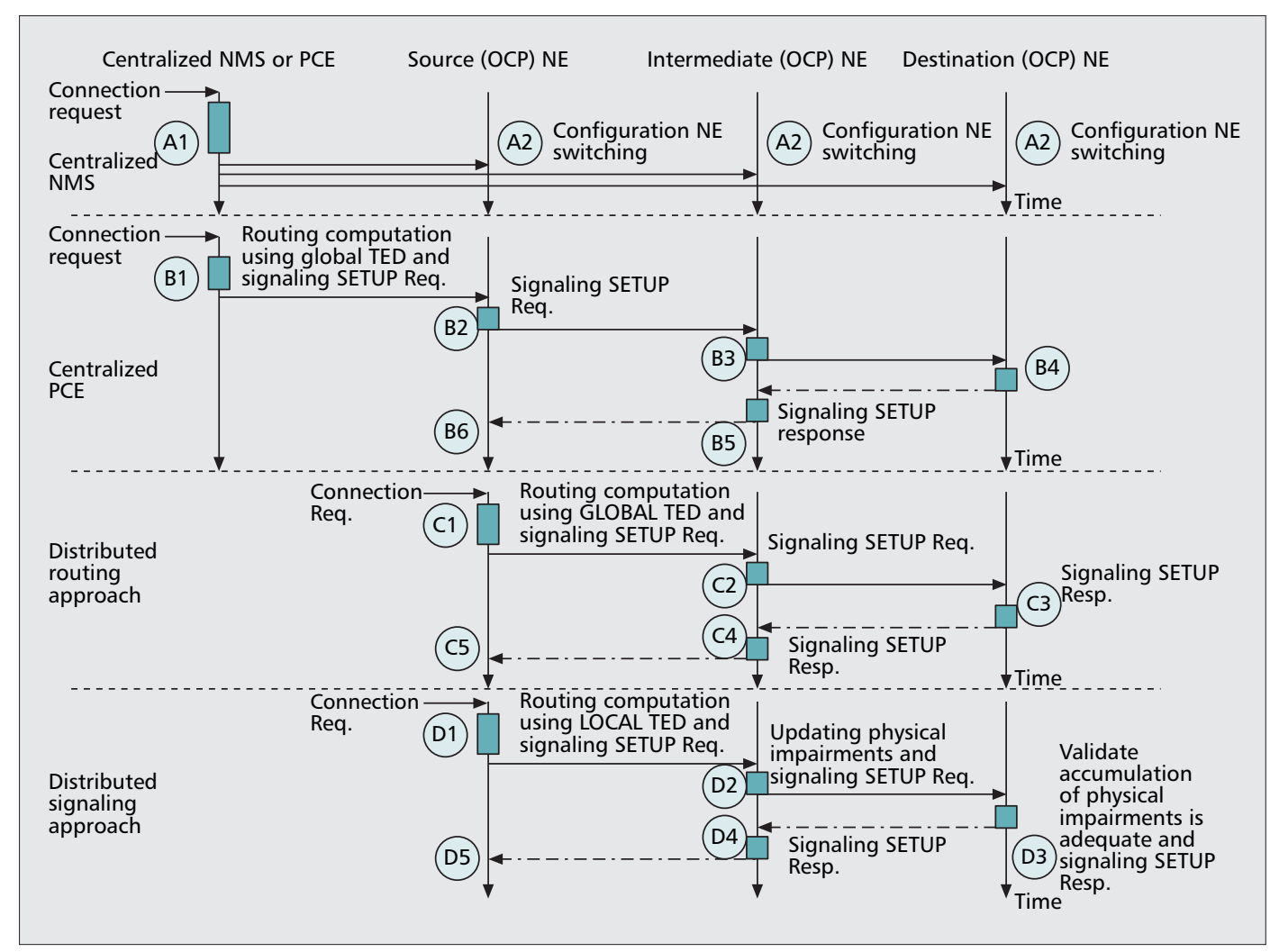

The main advantage
of the centralized
NMS-based
approach is that it is
aware of the
complete, detailed,
and periodical view
of the whole
network
(i.e., available
resources, topology,
physical impairments)
through a centrall
global database.

Figure 3. Flow diagram of different impairment-aware lightpath setup approaches.

link. As shown in Fig. 3, when the connection request arrives to the source $\mathrm{NE}$, first the RWA algorithm (step C1) is executed, taking into consideration its own TED and the potential constraints required by the connection. The on-line RWA algorithm computes a route considering network performance objectives (e.g., efficient network resource utilization) and the required end-to-end optical signal quality. The computed route is then passed to GMPLS signaling protocol (e.g., Resource Reservation Protocol with traffic engineering, RSVP-TE), which allocates the resources to set up the requested lightpath (steps $\mathrm{C} 2-\mathrm{C} 5$ ). If the requirements cannot be satisfied, the connection is blocked.

In the distributed signaling approach, existing GMPLS signaling protocols such as RSVPTE are extended to perform on-line dynamic estimation of the signal quality during the lightpath setup process [8]. In this approach no extensions are introduced in the current routing protocols. Thus, the routing algorithm (i.e., RWA) computes routes from an optimal network performance perspective by using only TE link parameters maintained in the TED (step D1). Some modifications are introduced in the currently used GMPLS signaling protocol to collect signal impairments that characterize the traversed links from the source to the destination along the SETUP message (Fig. 3). Thus, every traversed NE, before propagating the SETUP message, updates these parameters (step D2) by adding its own local values (i.e., ongoing link information). Admission control at the destination checks whether the overall accumulated parameter values on the receiver interface are compliant with an acceptable range of required QoS (step D3). If the accumulated transmission impairments do not exceed such a range, the connection is established and the SETUP response is sent back to the source node (steps D4 and D5). Otherwise, the lightpath is refused and further setup attempts following different routes are triggered to serve the connection. Lightpath setup evaluation (and rejection) at intermediate nodes and crankback schemes can also be activated. As a result, this mechanism allows guaranteeing the required signal-quality at the destination node along the complete route.

\section{DISCUSSION AND ASSESSMENT OF APPROACHES}

This section presents advantages and drawbacks of the aforementioned approaches for impairment-aware lightpath set up, as summarized in Table 2.

The main advantage of the centralized NMS-based approach is that it is aware of the complete, detailed, and periodical view of the whole network (i.e., available resources, topology, physical impairments) through a central/global database. Therefore, under the only constraint of the noninstantaneous database update time, all the path requests can be optimally served, while the potential effect on the signal quality of existing connections is taken into account. However, in the centralized approach interoperability and scalability problems, as well as significant technical hitches 


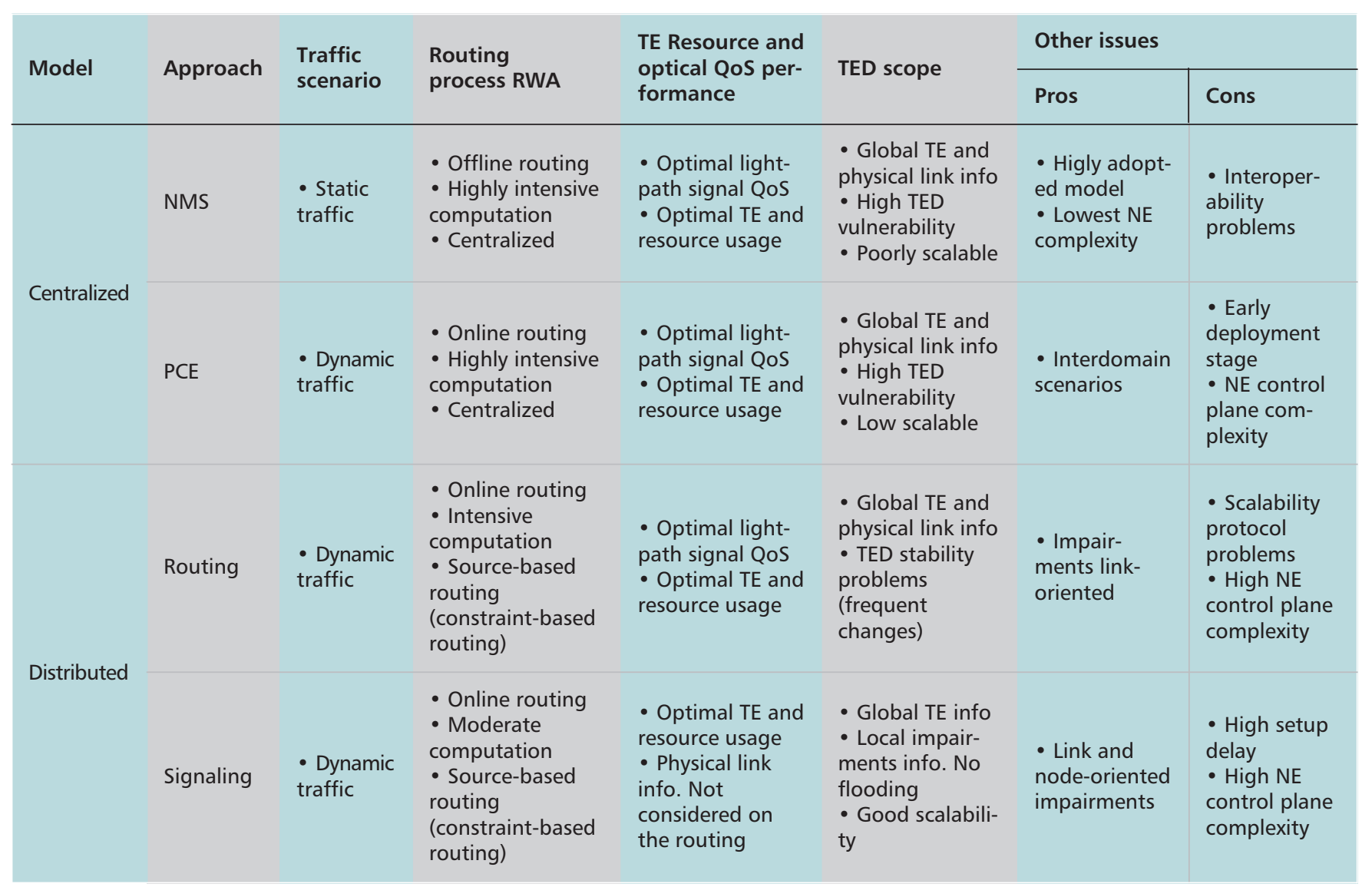

Table 2. Comparison of the impairment-aware lightpath set up approaches.

such as real-time lightpath provisioning, automatic network topology, and resource discovery, as well as fast connection recovery, can arise when dealing with services carrying timesensitive traffic due to its own intrinsic nonautomatic operation.

The centralized PCE approach with distributed lightpath provisioning provides automatic establishment of optical connections and network topology discovery. Moreover, since all the paths within the domain are computed by the PCE, a lightpath provisioning that optimizes both resource usage and optical signal quality can be reached. However, the lack of flexibility together with the scalability problems are the main drawbacks of the PCE approach. Indeed, rapid restoration in case of (multiple) failures cannot be achieved because the central (PCE) node needs to manage the isolation of the failure, recomputation of all failed routes, and upload of all the routes to every node involved in the restoration. In addition, scalability and vulnerability to database failure represent fundamental and critical issues that are difficult to handle with a single routing element. Finally, PCE standardization is still at an early stage and its applicability is so far mainly devoted to inter-domain path computation instead of route calculation within a single (transparent) domain.

In the distributed routing approach, a GMPLS routing protocol (e.g., OSPF-TE) is used to populate link information involving TE and physical performance characteristics. Only minor protocol changes are expected to enhance current routing protocols to take into account linear physical impairments. One of the most important benefits with this solution is that new RWA algorithms can be proposed for dealing jointly with signal quality and optimal network resource utilization (e.g., bandwidth). However, this approach introduces some limitations that are mainly due to the essence of the routing protocol, such as TED inconsistency, scalability, and stability problems when the link information changes (e.g., physical parameters) are frequent. An example of this limitation is the variation of the power levels in amplified WDM transmission systems due to dynamic setup and teardown of channels. If channels are equalized by adjusting the transmitting powers at the source node based on information (e.g., OSNR) conveyed from the receiving interface [9], several iterations may be required to achieve equalization, which may result in both excessive flooding and signal quality oscillations of already established and affected connections. Therefore, the distributed routing approach seems to be more plausible when the variability of physical-layer parameters is moderate. Concerning the routing computation process, a multiconstrained path computation (RWA algorithm) is required for achieving both optimal network performance and sufficient quality of the optical signal, which results in an NP-hard problem. As a consequence, this problem heavily impacts the load of the NE's processing unit (CPU), which might result in large computation time [10]. 


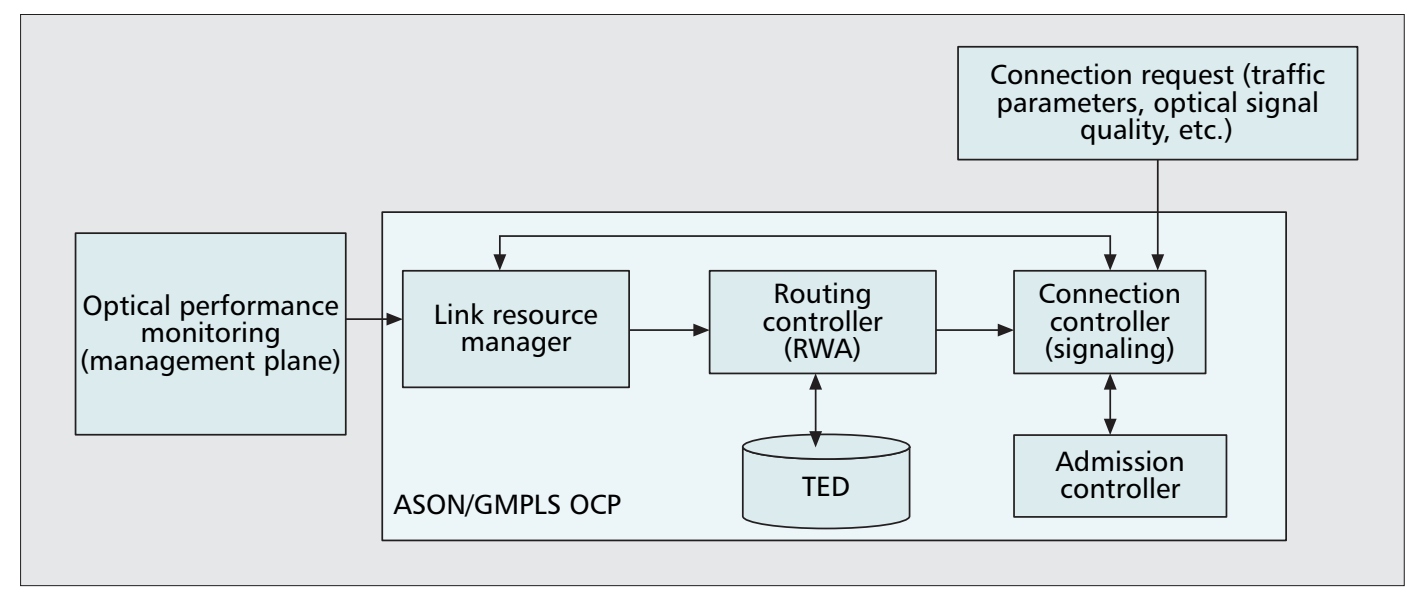

Figure 4. Architecture of a distributed ASON/GMPLS impairment-aware optical control plane (OCP).

The distributed signaling approach does not introduce any global flooding of physical impairment information. The information is maintained just locally on each NE. Thus the main benefit of the distributed signaling approach is that it can easily handle frequent changes of the physical parameters within the transparent network, such as the equalization procedure described above. Moreover, this approach can be utilized without increasing the node's CPU load and the computational delay, due to the independence from the physical information variability. However, the signaling-based approach is also subject to some drawbacks. The possible failure of the first setup attempt forces successive setup attempts, which increase both the overall amount of required signaling and the setup delay. Moreover, if the setup fails, in case of a forward resource reservation strategy, some resources may have been unnecessarily allocated. Finally, the physical-layer-unaware route computation performed in this approach can find suboptimal provisioning solutions and, particularly in the case of quasi-static physical parameters, may result in a less efficient utilization of network resources.

In summary, the centralized approach and the hybrid approach, although suffering some drawbacks, can be adopted as interim solutions, while the distributed approaches, further detailed in the following section, appear as a future-proof solutions.

\section{REQUIREMENTS FOR IMPLEMENTING DISTRIBUTED IMPAIRMENT-AWARE Control Plane}

In this section we outline the extensions to the ASON and GMPLS standards [1, 2] required to implement impairment-aware RWA according to the distributed approaches described above. The requirements are expressed from two perspectives: the new architecture and functionalities that each OCP's element needs to perform, and the required physical-layer extensions to the control protocol (i.e., routing and signaling).

Figure 4 shows the ASON-based architec- ture of the impairment-aware control plane, which is constituted by four main elements, namely, the link resource manager (LRM), the connection controller (CC), the admission controller (AC), and the routing controller (RC). The LRM module [1] is the responsible for maintaining an updated view of all the local link resources present in the transport plane (e.g., link addressing, bandwidth, etc.). Aside from the TE attributes, it needs to be upgraded to support the physical link parameters, as listed in Table 1. This information, as depicted in Fig. 4, is delivered throughout the Network Management Interface [1], which connects the OPM, located at the management plane, and the LRM. Specifically, this interface defines the information flow that allows the LRM to acquire updated physical information, entailing a twofold problem: first, state the exact and necessary information (e.g., set $\mathrm{S}$ in Fig. 2b) required by a particular distributed approach; second, to design how the updating process is achieved without overloading the OCP. In [11], the authors present experimental results for measured delay when OPM updates performance parameters of a given physical link.

In the distributed signaling approach described above, the $\mathrm{CC}$ element defined in [1] needs to be enhanced to provide a set of mechanisms to estimate the signal quality locally during the lightpath setup and to manage setup re-attempts effectively in case of inadequate signal quality at either the intermediate node or the destination. In [8], the authors proposed and evaluated these new required $\mathrm{CC}$ functionalities. In particular, the AC ASON control plane's element [1] is responsible for informing the $\mathrm{CC}$ about whether the accumulated optical parameters are admissible by the connection being established. Therefore, if the accumulated parameters exceed an admissible value or threshold, further re-attempts shall try to follow a different path to set up the requested lightpath. Finally, the RC performs the source-initiated route computation for each requested lightpath by means of RWA algorithms. While in the distributed signaling approach these RWA algorithms only consider TE constraints and
While in the distributed signaling

approach these RWA algorithms only

consider TE constraints and

network resources, in the distributed routing approach new impairmentaware RWA algorithms shall be proposed for computing feasible end-to-end routes considering both TE and optical physicallink constraints. 
This study shows

that in a dynamic

traffic scenario

wherein physical-

layer parameters may

vary frequently,

distributed control-

plane-based

approaches are

found to be more

suitable for efficiently

managing the

provisioning of fast

quality-enabled

connections than

centralized models. network resources, in the distributed routing approach new impairment-aware RWA algorithms shall be proposed for computing feasible end-to-end routes considering both TE and optical physical-link constraints [3].

Besides the presented enhancements to the OCP architecture, the two discussed distributed approaches require specific control-protocol extensions to perform the impairment-aware RWA. On one hand, in the distributed routing approach, current GMPLS routing protocols (e.g., OSPF-TE) need to be extended to flood updated physical-link information within the transparent domain [6]. Similarly, as TE attributes (e.g., bandwidth change), once a node's LRM detects a variation of the physical link information monitored by the OPM, new traffic engineering link-state advertisements (TE-LSAs) [2], carrying both TE and the updated physical-link parameters, should be generated by that node, and then disseminated to the whole network. This new extended physical-link information is encapsulated within the top-level link type, length, value (TLV) as regular TE-based sub-TLVs to carry physical link information specified in Table 1 (e.g., noise power $N_{k}$ parameter, etc.). Upon receiving a new TE-LSA, the carried information is then collected at every node (TED), and used by the impairment-aware RWA algorithm. In [3], the authors proposed an impairment-aware RWA, wherein the route computation is split into two separated steps: first to obtain a route only considering TE or network-layer attributes, and then to validate the optical signal quality along that computed path, using the physical link constraints (i.e., PMD and OSNR constraints).

In the distributed signaling approach, on the other hand, physical link information is to be included in the current GMPLS signaling protocols. Thus, in contrast to the distributed routing approach, this information is not to be disseminated to the entire network, but locally stored on every OCP LRM and requested by the CC (impairment-aware signaling) when the lightpath setup process is passing a particular NE. In [8]. the authors proposed proper extensions (i.e, objects) to GMPLS RSVP-TE Path and PathError messages, in order to convey attenuation parameter as a physical link parameter, estimate the quality of received optical signal, and notify potential path rejection due to physical parameters being out of an acceptable range, respectively.

\section{CONCLUSIONS}

In this article the most significant physical impairments that may be relevant for transparent WDM networks have been singled out and some optical performance measurements techniques have been presented. The problem of taking into account these physical impairments in the RWA procedure and lightpath provisioning has been tackled with proposed solutions to be implemented at the management plane or control plane level.

A fully centralized (NMS-based), a hybrid (PCE-based), and two distributed (routing- and signaling-based) approaches to establish lightpaths with guaranteed optical signal quality at the receiver have been presented and assessed. This study shows that in a dynamic traffic scenario wherein physical-layer parameters may vary frequently, distributed controlplane-based approaches are found to be more suitable for efficiently managing the provisioning of fast quality-enabled connections than centralized models. Nevertheless, the centralized and hybrid models can be helpful as migration solutions towards the distributed models or as medium-term solutions for transparency domains with a moderate number of nodes.

An experimental evaluation of the presented distributed approaches is planned by properly upgrading the intelligent all-optical ADRENALINE testbed [12] within the framework of the IST Network of Excellence e-Photon/ONe + . In particular, the goal of these implementation and experimentation tests is to validate the feasibility of the proposed distributed approaches as well as to asses the correctness of the adopted model when only considering linear impairment effects.

\section{ACKNOWLEDGMENTS}

This work was partially funded by the MEC (Spanish Ministry of Science and Education) through the project RESPLANDOR under contract TEC2006-12910/TCM; on the Italian side, partially by the MIUR (Ministry of University and Research) though the GRID.IT project under contract RBNE01KNFP; and developed within the framework of both COST270 WG1 and Network of Excellence ePhoton/ONe.

\section{REFERENCES}

[1] ITU-T Rec. G.8080/Y.1304, "Architecture for the Automatically Switched Optical Network (ASON)," Nov. 2001.

[2] E. Mannie et al., "Generalized Multi-Protocol Label Switching (GMPLS) Architecture," IETF RFC 3945, Oct. 2004.

[3] Y. Huang, J. P. Heritage, and B. Mukherjee, "Connection Provisioning with Transmission Impairment Consideration in Optical WDM Networks with High-Speed Channels," IEEE/OSA J. Lightwave Tech., vol. 23, no. 3, , Mar. 2005, pp. 982-93.

[4] D. C. Kilper et al., "Optical Performance Monitoring," IEEE/OSA J. Lightwave Tech., vol. 22, no. 1, Jan. 2004, pp. 294-304.

[5] ITU-T Draft new Recom. G.697, "Optical Monitoring for DWDM Systems," June 2004.

[6] J. Strand, A. L. Chiu, and R. Tkach, "Issues for Routing in the Optical Layer," IEEE Commun. Mag., vol. 39, no. 2, Feb. 2001, pp. 81-87.

[7] A. Farrel, J. P. Vasseur and J. Ash, "A Path Computation Element (PCE)-Based Architecture," IETF RFC 4655, Aug. 2006.

[8] F. Cugini et al., "Physical Impairment Aware Signaling for Dynamic Lightpath Set Up," Proc. 31st Euro. Conf. Optical Commun., Glasgow, U.K., Sept. 2005.

[9] A. R. Chraplyvy, J. A. Nagel, and R. W. Tkach, "Equalization in Amplified WDM Lightwave Transmission Systems," IEEE Photonics Tech. Lett., vol. 4, no. 8, Aug. 1992, pp. 920-22.

[10] A. Jukan and G. Franzl, "Path Selection Methods with Multiple Constraints in Service-Guaranteed WDM Networks," IEEE/ACM Trans. Net., vol. 12, no. 1, Feb. 2004.

[11] C. Pinart, R. Martinez and G. Junyent, "Experimental Implementation of Dynamic in-Service Performance Monitoring for Lambda Services," Proc. 31st Euro. Conf. Optical Commun., Glasgow, U.K., Sept. 2005.

[12] R. Munoz et al., "The ADRENALINE Testbed: Integrating GMPLS, XML and SNMP in Transparent DWDM Network," IEEE Commun. Mag., vol. 43, no. 6, Aug. 2005 pp. S40-S48. 


\section{BIOGRAPHIES}

RICARDO MARTINEZ (ricardo.martinez@cttc.es) is a graduate in telecommunications engineering of the Polytechnic University of Catalonia (UPC, 2002). He is currently pursuing a Ph.D. degree in the Department of Signal Theory and Communications, UPC, under the supervision of Prof. Grabriel Junyent. Since May 2005 he has been a research assistant in CTTC's Optical Networking Area. His research interests include provisioning, survivability, and reliability within photonic networks as well as GMPLS architecture and protocols.

CAROLINA PINART (carolina.pinart@cttc.es) is a graduate in telecommunications engineering with a Ph.D. from UPC, April 1999 and December 2005, respectively. She joined CTTC in November 2001 as director of institutional relations, collaborating as well with CTTC's Optical Networking Area. Prior to joining CTTC, she served as an undergraduate researcher (Center for Research SiemensNixdorf, Munich, Germany, 1998-1999) and as a technology consultant in wireless and in-vehicle systems and networks (Altran Group, Paris, France, 1999-2001). In summer 2006 she was an invited researcher at the National Institute of Information and Communications Technology (NICT), Tokyo, Japan. Since 2000 she has been a participant in 11 Spanish and European (ESPRIT, EUREKA, and IST programmes) R\&D projects in wireless and optical networking. She has published six papers in journals and magazines, and more than 25 papers at conferences. She was awarded a 2003 Graduate Prize of the Fundació Agrupació Mútua and a 2006 Post-Doctoral Fellowship of the Japan Society for the Promotion of Science.

FILIPPO CUGINI (filippo.cugini@cnit.it) received a Laurea degree in telecommunication engineering from the University of Parma, Italy. Since 2001 he has been a research engineer at the CNIT National Laboratory of Photonics Networks, Pisa, Italy. His main research interests include MPLS and GMPLS protocols and architectures, survivability in IP over WDM networks, and traffic engineering in grid networking.

NicolA ANDRIOLLI (nick@sssup.it) holds a Laurea degree in telecommunications engineering (2002) from the University of Pisa, Italy, and a Diploma degree (2003) from Scuola Superiore Sant'Anna, Pisa, Italy. He is currently working toward a Ph.D. degree at Scuola Superiore Sant'Anna. He was a visiting student at both BUTE, Budapest, Hungary, and COM•DTU, Copenhagen, Denmark, and a visiting trainee at NICT, Tokyo, Japan. His research interests include wavelength routed networks design, QoS and fault tolerance in optical networks, GMPLS architecture, and optical packet switching.

LUCA VALCARENGHI (luca.valcarenghi@sssup.it) holds a Laurea degree in electronics engineering (1997) from the Politecnico di Torino, Italy, an M.S. degree in electrical engineering (1999), and a Ph.D. degree in electrical engineering-telecommunications (2001) both from the University of Texas at Dallas (UTD). Between January 2002 and August 2002 he was a research associate at the Optical Networking Advanced Research (OpNeAR) Laboratory of the University of Texas at Dallas Erik Jonsson School of Electrical Engineering and Computer Science. Since September 2002 he has been an assistant professor at the Scuola Superiore Sant'Anna of University Studies and Doctoral Research, Pisa, Italy. His main research interests are optical networks design, analysis, and optimization; artificial intelligence optimization techniques; communication networks reliability; IP over WDM networking; QOS in network infrastructures for grid computing; and broadband wireless access (802.16).

PIERO CASTOLDI (castoldi@sssup.it) is currently an associate professor of telecommunications at the Center of Excellence for Communication Networks Engineering of Scuola Superiore Sant'Anna, Pisa, Italy. He is also director of the CNIT National Laboratory of Photonic Networks, Pisa, Italy. His scientific activity has covered the areas of wired and wireless networks and, more recently, design, resilience schemes, and service platforms for metro and core networks. He has authored more than 80 technical papers and a book on CDMA.
LENA WoLSINSKA [M] (lena@it.kth.se) received her M. Sc. degree in electrical engineering from the Warsaw Institute of Technology, Poland, and her Ph.D. degree in photonics from the Royal Institute of Technology, Stockholm, Sweden. She joined the Royal Institute of Technology in 1986 where she is currently an associate professor at the School of Information and Communication Technology (ICT). Her research interests are in the areas of photonics in switching and management of optical networks. Currently she is working on optical packet switching, IP over WDM networking, and optical network reliability and survivability aspects.

JAUME COMELLAS (comellas@tsc.upc.edu) received M.S (1993) and Ph.D. (1999) degrees in telecommunications Engineering from UPC. Since 1992 he has been a staff member of the Optical Communications Research Group of UPC. His current research interests mainly concern optical transmission and IP over WDM networking topics. He has participated in different research projects funded by the Spanish government and the European Commission. He has coauthored more than 100 research articles in national and international journals and conferences. He is an associate professor and currently head of laboratories at the Signal Theory and Communications Department of UPC. Currently, he is also involved in the Advanced Broadband Communications Center.

GABRIEL JUNYENT (junyent@tsc.upc.edu) is a telecommunications engineer (Universidad Politécnica de Madrid, UPM, 1973), and holds a Ph.D. degree in communications (UPC, 1979). He has been a teaching assistant (UPC, 1973-1977), adjunct professor (UPC, 1977-1983), associate professor (UPC, 1983-1985), and professor (UPC, 1985-1989), and has been a full professor since 1989. In the last 15 years he has participated in more than 30 national and international R\&D projects, and has published more than 30 journal papers and book chapters and 100 conference papers. 